San Jose State University

SJSU ScholarWorks

Faculty Publications, Chemistry

Chemistry

$1-1-1982$

\title{
Measurement of Synchrotron x-ray energies and line shapes using diffraction markers
}

Juana Vivó Acrivos

San Jose State University, juana.acrivos@sjsu.edu

S.S. P. Parkin

San Jose State University

K. Hathaway

San Jose State University

J. R. Reynolds

San Jose State University

M. P. Klein

University of California, Berkeley

See next page for additional authors

Follow this and additional works at: https://scholarworks.sjsu.edu/chem_pub

Part of the Physical Chemistry Commons

\section{Recommended Citation}

Juana Vivó Acrivos, S.S. P. Parkin, K. Hathaway, J. R. Reynolds, M. P. Klein, A. Thompson, and D. Goodin. "Measurement of Synchrotron x-ray energies and line shapes using diffraction markers" Review of Scientific Instruments (1982): 575-581. https://doi.org/10.1063/1.1137026

This Article is brought to you for free and open access by the Chemistry at SJSU ScholarWorks. It has been accepted for inclusion in Faculty Publications, Chemistry by an authorized administrator of SJSU ScholarWorks. For more information, please contact scholarworks@sjsu.edu. 
Authors

Juana Vivó Acrivos, S.S. P. Parkin, K. Hathaway, J. R. Reynolds, M. P. Klein, A. Thompson, and D. Goodin 


\section{AIP}

\section{Measurement of synchrotron xray energies and line shapes using diffraction markers}

J. V. Acrivos, K. Hathaway, J. Reynolds, J. Code, S. Parkin et al.

Citation: Rev. Sci. Instrum. 53, 575 (1982); doi: 10.1063/1.1137026

View online: http://dx.doi.org/10.1063/1.1137026

View Table of Contents: http://rsi.aip.org/resource/1/RSINAK/v53/i5

Published by the American Institute of Physics.

\section{Related Articles}

ECE-imaging of the $\mathrm{H}$-mode pedestal (invited)

Rev. Sci. Instrum. 83, 10E329 (2012)

Response to "Comment on 'Scalings for radiation from plasma bubbles"' [

Phys. Plasmas 18, 034701 (2011)

]

Phys. Plasmas 18, 034702 (2011)

Betatron $\mathrm{x}$-ray generation from electrons accelerated in a plasma cavity in the presence of laser fields

Phys. Plasmas 16, 103103 (2009)

Dynamics of emitting electrons in strong laser fields

Phys. Plasmas 16, 093115 (2009)

Undulator radiation in a periodic magnetic field with a constant component

J. Appl. Phys. 104, 124507 (2008)

\section{Additional information on Rev. Sci. Instrum.}

Journal Homepage: http://rsi.aip.org

Journal Information: http://rsi.aip.org/about/about_the_journal

Top downloads: http://rsi.aip.org/features/most_downloaded

Information for Authors: http://rsi.aip.org/authors

\section{ADVERTISEMENT}

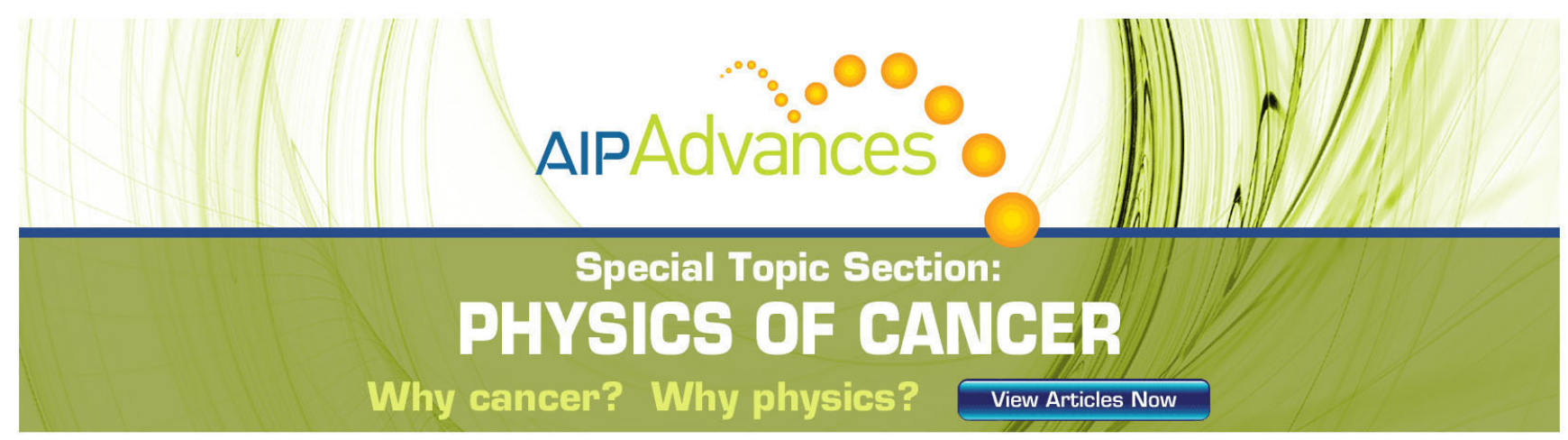




\title{
Measurement of synchrotron x-ray energies and line shapes using diffraction markers
}

\author{
J. V. Acrivos, K. Hathaway, J. Reynolds, J. Code, and S. Parkin \\ San José State University, San José, California 95192 \\ M. P. Klein, A. Thompson, and D. Goodin \\ Laboratory of Chemical Biodynamics, Lawrence Berkeley Laboratory, University of California, Berkeley, \\ California 94720
}

(Received 20 August 1981; accepted for publication 18 January 1982)

Standard reference markers for accurate, reproducible synchrotron $\mathrm{x}$-ray energies are obtained using a three $\mathrm{Si}$ crystal spectrometer. The first two crystals are in the monochromator and the third is used to obtain diffraction markers which monitor the energy. Then for any value of the glancing angle on the reference Si crystal the energy for the (333) diffraction must occur at $3 / 4$ that of the (444) and $3 / 5$ of that for the (555). This establishes for the first time an absolute synchrotron energy scale. Higher-order diffractions are used to determine excitation line profiles. We conclude that the use of reference diffractions is necessary to measure reproducible $\mathrm{x}$-ray energies and to analyze the incident photons' line profile. The detection of diffractions near the edge of measurement and near the $\mathrm{Cu}$ edge will provide a fast secondary standard which will allow comparison of edge data between different laboratories. The diffraction profiles will allow the proper analysis of spectral line widths.

PACS numbers: $41.70 .+t$

\section{INTRODUCTION}

The accurate measurement of x-ray energies $E$ near $10^{4}$ $\mathrm{eV}$ to within $10^{2} \mathrm{ppm}$ is needed in order to ascertain the valence of the absorber in conductors and in biological materials because the valence is an indicator of how metal-insulator transitions and how biological red-ox reactions occur. In particular, we have correlated the metal-insulator transitions in the liquid metal $\mathrm{Rb}-\mathrm{NH}_{3}$ with the changes in the $\mathrm{x}$-ray absorption spectra near the $\mathrm{Rb} K$ edge. ${ }^{\prime}$ Here a single absorption near the edge (1s $\rightarrow \mathrm{np}, n \geq 4$ ) shifts as a function of the relative metal concentration by as much as $5 \mathrm{eV}$ as the valence changes from 0 to +1 . Also multiple valence has been deduced from shifts in the $\mathrm{X}$-ray absorption for SmS under pressure ${ }^{2}$ or by $Y$ substitution in $\mathrm{Sm}_{x} \mathrm{Y}_{1-x} \mathrm{~S}^{3}$ and by intercalation in $\mathrm{TaSe}_{2} .{ }^{4}$ The relative changes are easy to measure during a given experiment by running a set of standards together with the unknown samples. ${ }^{5}$ However, in order to compare data taken at different times, at different laboratories, it is important to measure the $a b$ solute value of the energy to within at least $10^{2} \mathrm{ppm}$. The purpose of this work is to describe a simple three $\mathrm{Si}$ crystal spectrometer shown in Fig. 1 which does the job.

The principles used to measure accurate wavelengths are described in Sec. II. ${ }^{6}$ The experimental details and error analysis for an example are applied in Sec. III to measurements of the $\mathrm{TaS}_{2}-\mathrm{N}_{2} \mathrm{H}_{4}$ and the $\mathrm{C}$ (graphite) $+\mathrm{AsF}_{5}$ systems carried out at the Stanford Synchrotron Radiation Laboratory (SSRL).

\section{X-RAY WAVELENGTHS}

Accurate $\mathrm{x}$-ray wavelength measurements are carried out using the Stenström correction to the Bragg relation. ${ }^{6}$ The Bragg relation is satisfied for the planes in the two Si crystals of the monochromator $(M)$ and for an additional reference crystal $(g)$ with respective scattering vectors $\mathbf{S}$ when:

$$
2 \sin \theta_{B} / \lambda=S,
$$

where $\theta_{B}$ is the angle which gives the correct wavelength $\lambda$ in air. Then if $\theta_{B}=\theta_{M B}$ at the monochromator crystals and $\theta_{B}=\theta_{g B}$ at a third reference crystal in the x-ray beam, a reference diffraction may be recorded when

$$
\sin \theta_{M B} / S_{M}=\sin \theta_{g B} / S_{g}=\lambda / 2=h c / 2 E .
$$

The value of $\theta_{B}$ is determined from the Darwin relation which corrects the glancing angle for the effects of the index of refraction. The $x$-ray glancing angle and the wavelength change from $(\theta, \lambda)$ in air to $\left(\theta^{\prime}, \lambda^{\prime}\right)$ within the crystal and are related by the real part of the index of refraction ${ }^{6}$ :

$$
1-\delta=\cos \theta / \cos \theta^{\prime}=\lambda / \lambda^{\prime} .
$$

The Stenström correction to the energy (due to $\delta$ ) in relations (2) is then

$$
\Delta E_{i} / E_{i}=-\delta_{i} / \sin ^{2} \theta_{i}=\Delta_{i s},
$$

where $i=g$ or $M$ and near the $\mathrm{Cu} K$ edge $\left(\delta=\delta_{0}=7.3\right.$ 


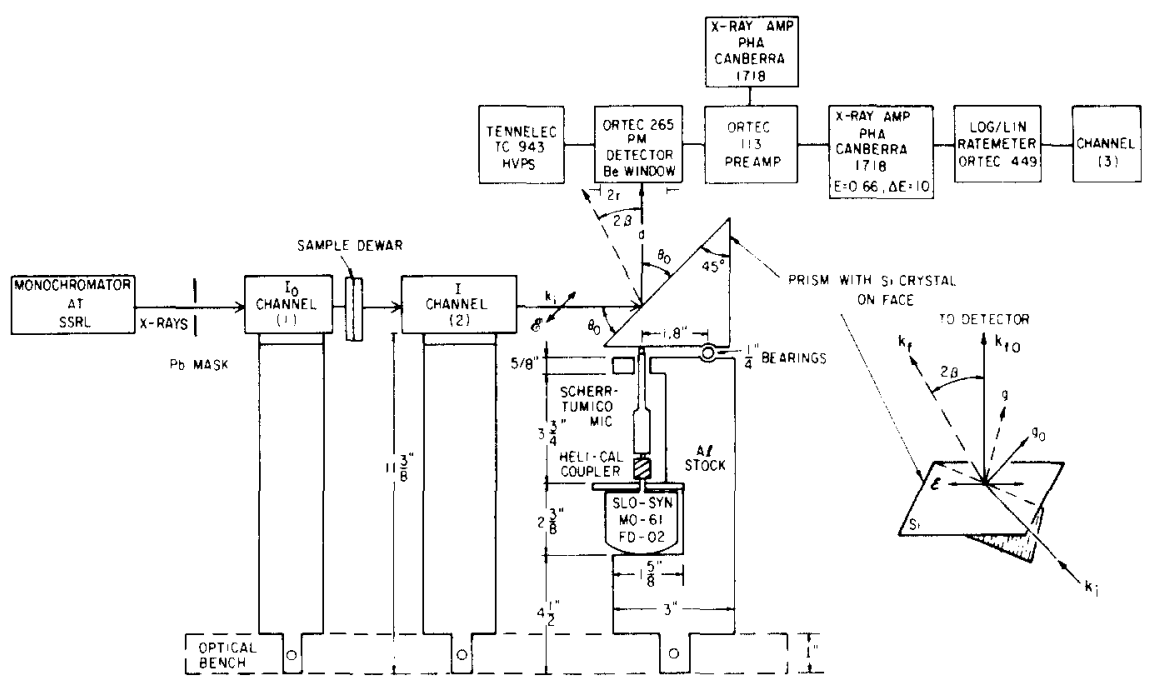

FIG. 1. Block diagram of the experimental configuration used to introduce reference markers as SSRL.

$\times 10^{-6}$ and $\theta_{B} \approx 21^{\circ}$ ) this is of the order of $60 \mathrm{ppm}$ or if $\theta$ is the measured value ${ }^{6}$ :

$$
\theta_{B}=\theta-2 \delta \operatorname{csec} 2 \theta .
$$

Thus to evaluate the energy to within an accuracy of $10^{2}$ ppm, one needs to know the glancing angle $\theta$ to within the same accuracy. If $\theta$ can be measured directly the solution is obvious. ${ }^{6}$ The situation with synchrotron sources (e.g., at SSRL), however, is less direct. There the monochromator is driven by a stepping motor $(M$ step) with a known incremental angle per step $\left(r_{\theta_{\mu}}\right)$, but the absolute angle is unknown and must be determined by a calibration (e.g., at a known energy). We may write:

$$
\theta=\theta_{M}+c_{M} \text { where } \theta_{M}=r_{\theta_{M}}(M \text { steps })
$$

and $c_{M}$ is an offset angle to be determined by a calibration. The correct energy selected by the monochromator crystal is then given by

$$
E_{M}=h c S_{M}\left[1+\delta / \sin ^{2}\left(\theta_{M}+c_{M}\right)\right] / 2 \sin \left(\theta_{M}+c_{M}\right) \text {. }
$$

In a conventional two $\mathrm{Si}$ crystal spectrometer, $c_{M}$ is calibrated by identifying the reported edge energy $E_{0}$ with some reasonable feature of the metal x-ray absorption near the $\mathrm{Cu} K$ edge. Some users identify the first inflection point with $E_{0}(8.9803 \mathrm{keV})$ and one assumes that $\delta$ varies linearly with $\lambda^{2,6}$; i.e., the Stenström relation (4) is a constant. However, near the $\mathrm{Fe} K$ edge this calibration of $c_{M}$ places the first inflection point $2 \mathrm{eV}$ above $E_{0}$ $(7.1112 \mathrm{keV})$. This is not possible because the true edge is usually preceded in energy by some white line features. Other users place $E_{0}$ near the first sharp feature (minimum or maximum) of the extended $\mathrm{x}$-ray absorption fine structure (EXAFS) which is ambiguous but much more plausible than the first inflection point. Also internal standards can be used to provide relative energy measurements. ${ }^{5}$ However, an absolute energy calibration requires a three-crystal spectrometer. Figure 1 describes the first one used successfully at SSRL, but the simple apparatus can be used at any other synchrotron source.

The only variable in the optical system shown in Fig.
1 is the glancing angle $\theta_{0}$ of $\mathrm{x}$ rays (coming through counter I) on a Si crystal $\left(4 \times 10^{-4} \mathrm{~m}\right.$ thick $)$ cut along the (111) [or (110)] plane which is changed from $45^{\circ}$ by a step motor in order to place two or more diffraction markers near the edge being measured (Fig. 2) and two or more near the $\mathrm{Cu} K$ edge. The x-ray diffraction signal is recorded on a third (fluorescence) channel. The energy calibration is carried out by generating tables of $E_{g}$ vs $\theta_{0}\left(\theta_{0}=40^{\circ}-45^{\circ}\right.$ in increments of $\left.0.1^{\circ}\right)$ using relations (2) and (6) and carrying out three additional edge measurements at the beginning and end of every period, during which $\theta_{0}$ is left unchanged. First, the $\mathrm{Cu} K$ edge is run. The strong diffractions near the $\mathrm{Cu} K$ edge shown in Fig. 2 are for $g=(333)$ and (335). These are easily identified and used to determine $\theta_{0}$ to within $100 \mathrm{ppm}$ by interpolating from the tables. Then the (444) diffraction near the As $K$ - or Ta $L_{I}$ edge is used to ascertain $c_{M}$ from the fact that $E_{333} / E_{444}=3 / 4$. Finally, the edge being investigated is scanned and the strong diffractions predicted by the tables are assigned. The procedure requires only three extra scans for calibration and the wealth of diffractions for the Si crystal allows one to place a diffraction marker or two near the edge being measured. The diffraction markers are then used to check the stability of the calibration throughout an entire experiment as long as care is exercised to maintain $\theta_{0}$ constant.

The reference relation (2) is written for the crystallographic planes normal to $g=(h k l)$ in the reference marker using the crystal Cartesian axes $\left(Z\left\|g_{0}, X\right\| g_{x}\right.$, and $Y \| \epsilon$, the direction of the beam polarization in Fig. 3), i.e.,

$$
\begin{aligned}
S_{g} \sin \left(\theta_{M}\right. & \left.+c_{M}\right)=S_{M} \sin \theta_{0}\left[\mathbf{g} \cdot \mathbf{g}_{0} /\left(|g| \cdot\left|g_{0}\right|\right)\right. \\
& \left.+\cot \theta_{0} \mathbf{g} \cdot \mathbf{g}_{x} /\left(|g| \cdot\left|g_{x}\right|\right)\right]\left[1-\Delta_{g s}+\Delta_{M s}\right],
\end{aligned}
$$

where both $\mathbf{g}_{0}$ and $\mathbf{g}_{x}$ are first estimated by a Laue photograph but the exact orientation of the reference crystal in the laboratory axes must be obtained from $\left(2^{\prime}\right)$ for a series of calibration points. Also the value of $\theta_{0}$ for different $S_{g}$ diffractions has a specific dependence on $\theta_{M}$, 
a)

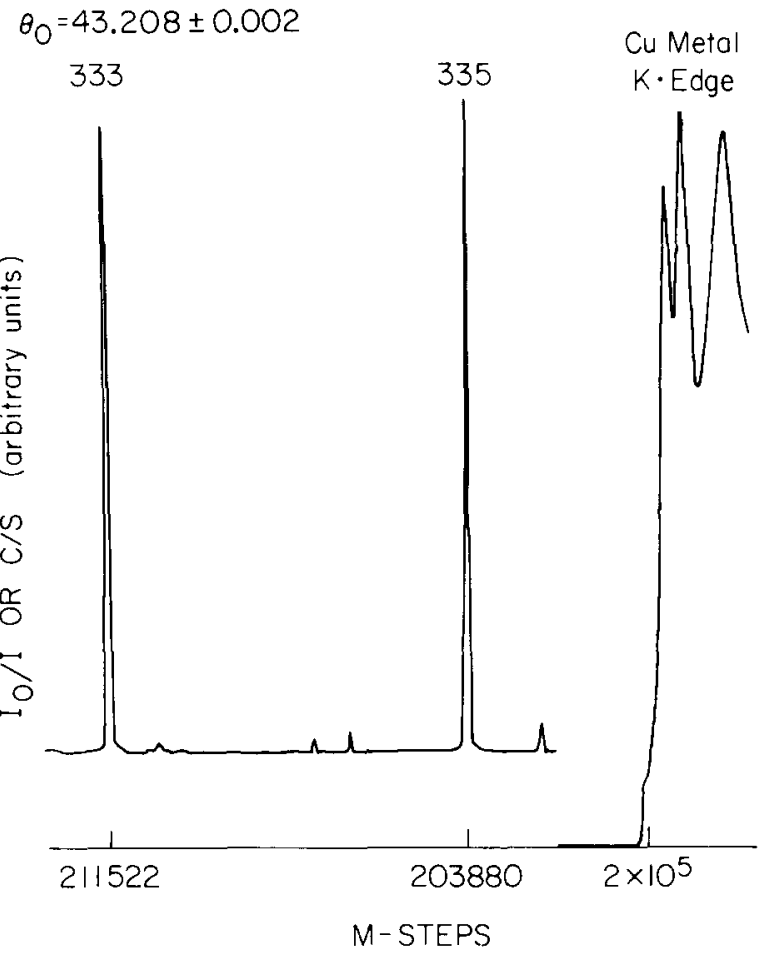

b) $\theta_{0}=43.208 \pm 0.002$

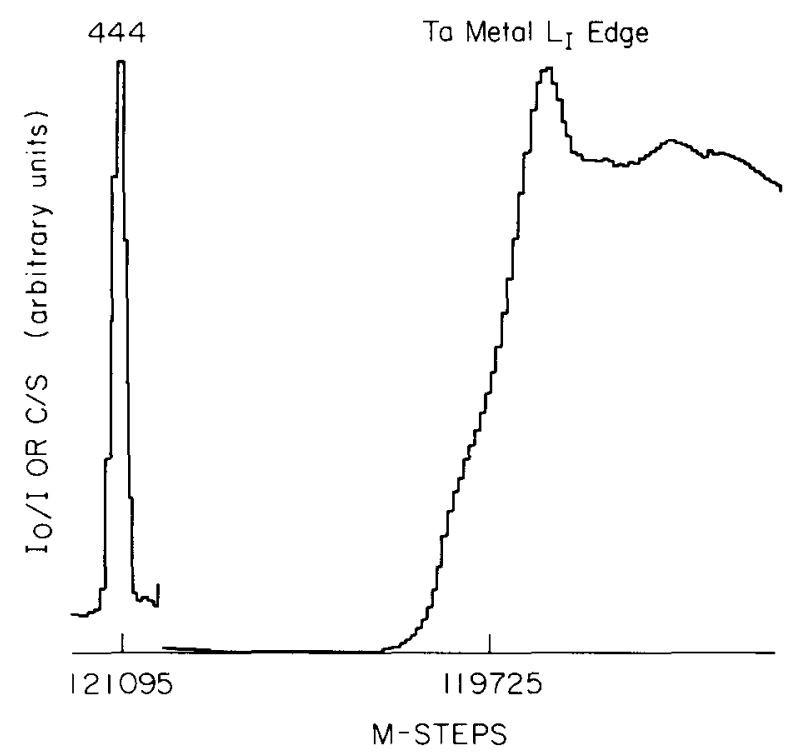

FIG. 2. Diffraction markers obtained with a Si crystal with $g_{0}=(111)$ and $g_{x}=(\overline{1} 12)$ in Fig. 3 ; (a) near the $\mathrm{Cu}-K$ and (b) $\mathrm{Ta}-L_{l}$ edges.

i.e., from ( $\left.2^{\prime}\right)$ it follows that a given $g$ diffraction tracks $\theta_{M}$ of the monochromator at the rate of

$$
\left(\frac{\partial \theta_{0}}{\partial \theta_{M}}\right)=\frac{\tan \left(A_{g}+\theta_{0}\right)}{\tan \left(\theta_{M}+c_{M}\right)}
$$

where $\tan A_{g}=\left|g_{0}\right| \mathbf{g} \cdot \mathbf{g}_{x} /\left[\left|g_{x}\right| \mathbf{g} \cdot \mathbf{g}_{0}\right]$ giving a change of energy with $\Delta \theta_{0}$ of:

$$
\Delta E / E=-\cot \left(A_{g}+\theta_{0}\right) \Delta \theta_{0}
$$

and from ( $\left.2^{\prime}\right)$ and (8) both $\theta_{0}$ and $A_{g}$ can be determined exactly. Figure 4 shows the dependence of $\theta_{0}$ on $\theta_{M}$ for the diffraction $g=(\overline{4} \overline{2} 6)$ in the orientation shown in Fig.
3 with $\mathbf{g}_{0}=(111)$ and $\mathbf{g}_{x}=(\overline{1} \overline{1} 2)$. The errors introduced by alignment are discussed in the Appendix. These do not affect the calibration. The relations (6) used to evaluate $c_{M}$ are

$$
\begin{aligned}
u_{3,4} & =4 E_{333} / 3 E_{444} \\
& =4 \sin \left(r_{\theta_{M}} M_{4}+c_{M}\right) / 3 \sin \left(r_{\theta_{M}} M_{3}+c_{M}\right)=1,
\end{aligned}
$$

and/or

$$
\begin{aligned}
u_{3,5} & =5 E_{333} / 3 E_{555} \\
& =5 \sin \left(r_{\theta_{M}} M_{5}+c_{M}\right) / 3 \sin \left(r_{\theta_{M}} M_{3}+c_{M}\right)=1,
\end{aligned}
$$

where the $M_{g}$ (monochromator) steps are experimental values measured when relation (2) is satisfied for the reference $g$ diffraction. Thus the relations $\left(6^{\prime}\right)$ calibrate the synchrotron radiation energy in the manner used earlier by both Stenström and Dumond. ${ }^{6}$

\section{EXAMPLE OF WORK DONE AT SSRL}

In the system shown in Fig. 1, the SLO-SYN motor rotates at the rate of $0.9^{\circ} / \mathrm{step}$ advancing the micrometer heat at the rate $r_{Z}=1.588 \times 10^{-6} \mathrm{~m} / \mathrm{step}$ causing a change in $\theta_{0}$ which is determined by the separation between the ball bearings supporting the back and front of the prism crystal mount of $4.58 \mathrm{~cm}$. Then the rate of change is $r_{\theta_{0}}=-3.47 \times 10^{-5} \mathrm{rad} / \mathrm{step}$, and the fractional rate of change in the energy which satisfies the Bragg condition for the $g$ plane follows from Eq. (8), i.e.,

$$
r_{E g}=(\Delta E / E) / \text { step }=-r_{\theta_{0}} \cot \left(A_{g}+\theta_{0}\right) / \text { step. }
$$

$k_{i}$ to $k_{f}$ DIFFRACTION

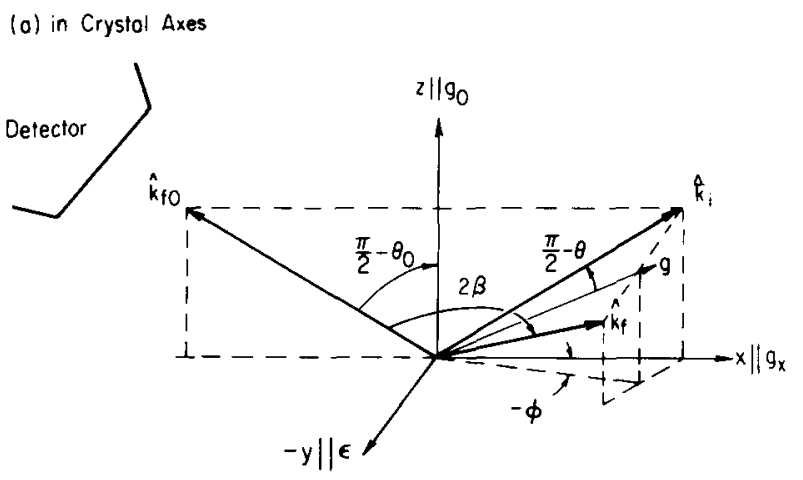

(b) in Laboratory Axes

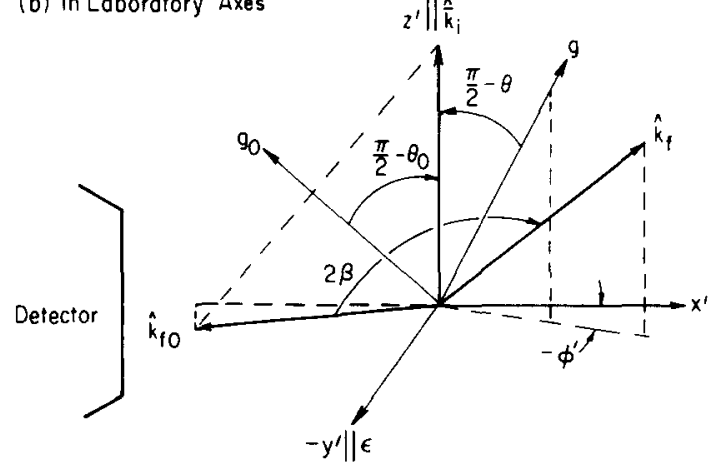

FIG. 3. Diffraction direction for a general crystal plane $g$. 


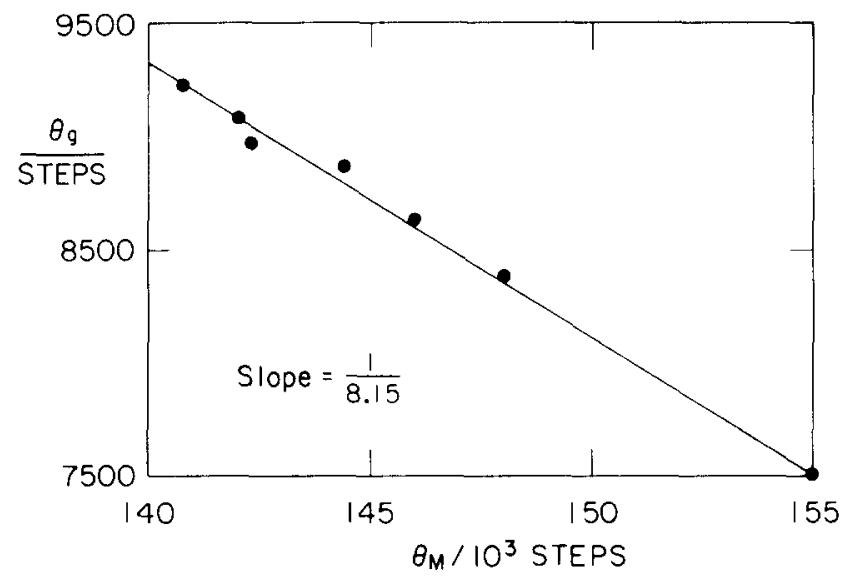

Fig. 4. Plot of $\theta_{0}$ /step vs $\theta_{M} / M$ step following the $g=(\overline{2} \overline{4} 6)$ diffraction (when $\mathbf{g}_{0}=(111)$ and $\mathbf{g}_{x}=(\overline{1} 12)$ in Fig. 3).

This is to be compared with the fractional rate of energy change per monochromator step:

$$
\begin{aligned}
r_{E_{M}} & =(\Delta E / E) / M \text { Step } \\
& =-r_{\theta_{M}} \cot \left(\theta_{M}+c_{M}\right) / M \text { Step, }
\end{aligned}
$$

where at the SSRL Station I-5 used in this work $r_{\theta_{M}}$ $=1.0908 \times 10^{-6} \mathrm{rad} / M$ step. The slope measured in Fig. 4 is in agreement with the calculated value in Table I:

$$
\begin{aligned}
r & =r_{E_{M}} / r_{E} \\
& =-3.144 \times 10^{-2} \tan \left(A_{g}+\theta_{0}\right) \cot \left(\theta_{M}+c_{M}\right)=-0.123,
\end{aligned}
$$

indicating that this diffraction marker can be moved at the rate of $0.28 \mathrm{eV}$ per step of the reference motor $(\mathrm{g}$ step) so that it can be placed at any desired energy to within a $25 \mathrm{ppm}$ accuracy. Then if $\theta_{0}$ is left unchanged during a set of measurements the absolute energy can be determined accurately by measuring say the (333), (444), and (555) diffractions. We have found that $\theta_{0}$ did remain constant for several days of running time but that any disturbance of the $\mathrm{Si}$ crystal prism mount can change $\theta_{0}$.

The energy calibration is carried out by minimizing the error in relations $\left(6^{\prime}\right)$ :

$$
\operatorname{DELU}_{i, j}=10^{6} \Delta u_{i, j}=10^{6} \mid 1-u_{i, j},
$$

versus $c_{m}$ as indicated in Fig. 6 and in Table I. The error propagated into $c_{m}\left(\Delta c_{m}\right)$ depends on the diffractions $i$, $j$; i.e.,

$$
\begin{aligned}
\Delta c_{m}= & \left\{\Delta u_{i, j}+\left|\theta_{M_{i}} \cot \theta_{M_{i} B}-\theta_{M_{j}} \cot \theta_{M_{j} B}\right| \Delta r_{\theta_{M}} / r_{\theta_{M}}\right. \\
& \left.+\left|\theta_{M_{i}} \cot \theta_{M_{i} B}\right| \Delta M_{i} / M_{i}+\left|\theta_{M_{i}} \cot \theta_{M_{i} B}\right| \Delta M_{j} / M_{j}\right\} \\
& \times\left\{\left|\cot \theta_{M_{j} B}-\cot \theta_{M_{i} B}\right|\right\}^{-1} \quad\left(10^{\prime}\right)
\end{aligned}
$$

\begin{tabular}{|c|c|c|c|c|c|c|c|}
\hline$M$-steps & & $\theta_{M B} / \mathrm{deg}$ & $E_{M} \mathrm{keV}$ & & & & \\
\hline \multicolumn{8}{|c|}{$E_{333}$ data (three measurements) } \\
\hline 211506 & & 21.8731 & 8.6670 & & & & \\
\hline \multicolumn{8}{|c|}{$E_{444}$ data (two measurements) } \\
\hline 121091 & & 16.2222 & 11.5581 & & & & \\
\hline \multicolumn{8}{|c|}{$E_{55 s}$ data (two measurements) } \\
\hline 68200 & & 12.9165 & 14.4449 & & & & \\
\hline \multicolumn{8}{|c|}{ Errors in ppm: Del $U_{3,4}=185$, Del $U_{3,5}=2$} \\
\hline \multicolumn{8}{|c|}{ Calibration data: $E_{0012 / 2}, \mathrm{E}_{333}, 1^{\text {st }}$ inflection points near $\mathrm{Fe}, \mathrm{Cu} K$ edges, $\mathrm{Ta} L \mathrm{III}$ and $L_{l}$ edges } \\
\hline $\begin{array}{l}308577 \\
203867 \\
294702 \\
200000 \\
168500 \\
119727\end{array}$ & & $\begin{array}{l}27.9401 \\
21.3957 \\
27.0729 \\
21.1540 \\
19.1853 \\
16.1369\end{array}$ & $\begin{array}{r}6.8913 \\
8.8510 \\
7.0945 \\
8.9474 \\
9.8255 \\
11.6175\end{array}$ & & & & \\
\hline \multicolumn{8}{|c|}{$\begin{array}{l}\text { Typical table of energies evaluated using relation (6) with } C_{M}=8.6540 \mathrm{deg} \\
\theta_{0}=43.1900 \mathrm{deg}, g_{0}=1.00 \quad 1.00 \quad 1.00, \quad g_{x}=-1.00-1.002 .00\end{array}$} \\
\hline$h$ & $k$ & $l$ & $E_{g} / \mathrm{keV}$ & $\theta_{g} / \mathrm{deg}$ & $M$-step $/ 10^{3}$ & $\begin{array}{c}\theta_{M B} / \\
\operatorname{deg}\end{array}$ & $d E_{\mathrm{g}} / d \theta_{\mathrm{0}} / \mathrm{eV} / \mathrm{deg}$ \\
\hline 0 & 0 & 12 & $2 \times 6.9156$ & 82.0744 & 306.8681 & 27.8333 & 16.8 \\
\hline 3 & 3 & 3 & 8.6670 & 43.1900 & 211.5032 & 21.8729 & -161.1 \\
\hline 3 & 3 & 5 & 8.8651 & 57.6101 & 203.2936 & 21.3598 & -98.2 \\
\hline 4 & 4 & 4 & 11.5560 & 43.1900 & 121.1381 & 16.2251 & -214.9 \\
\hline 5 & 5 & 5 & 14.4450 & 43.1900 & 68.1990 & 12.9164 & -268.6 \\
\hline
\end{tabular}

decreases as the denominator increases, giving a sharper minimum for $\Delta u_{3,5}$ than for $\Delta u_{3,4}$ vs $c_{M}$ in Fig. 6. Therefore we use $u_{3,5}$ for the calibration. Then under experimental conditions where the error in $\theta_{M_{i}}$ is $10 \mathrm{ppm}$ and the diffraction positions are known only to within the Stenström correction (60 ppm), we obtain an error $\Delta c_{M}$

TABLE I. Typical interpolation table used to determine $C_{M}=8.6540 \pm 0.002 \mathrm{deg}$. 
(a)

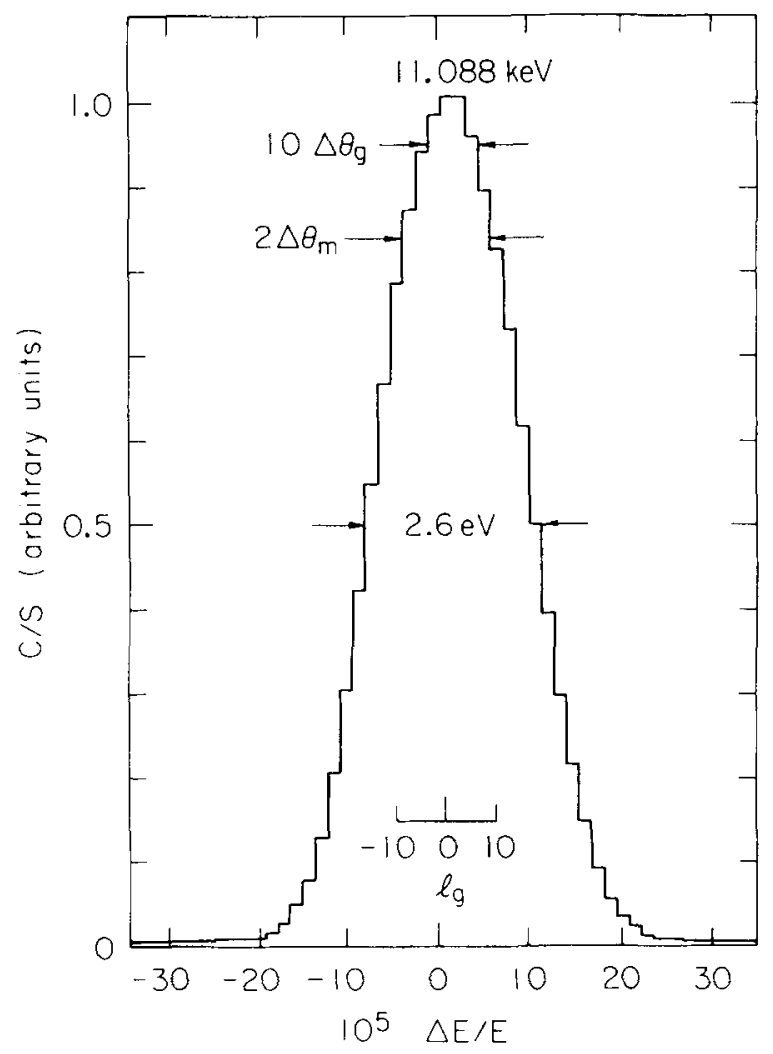

(b)

\section{$\theta=43.208 \pm 0.002$}

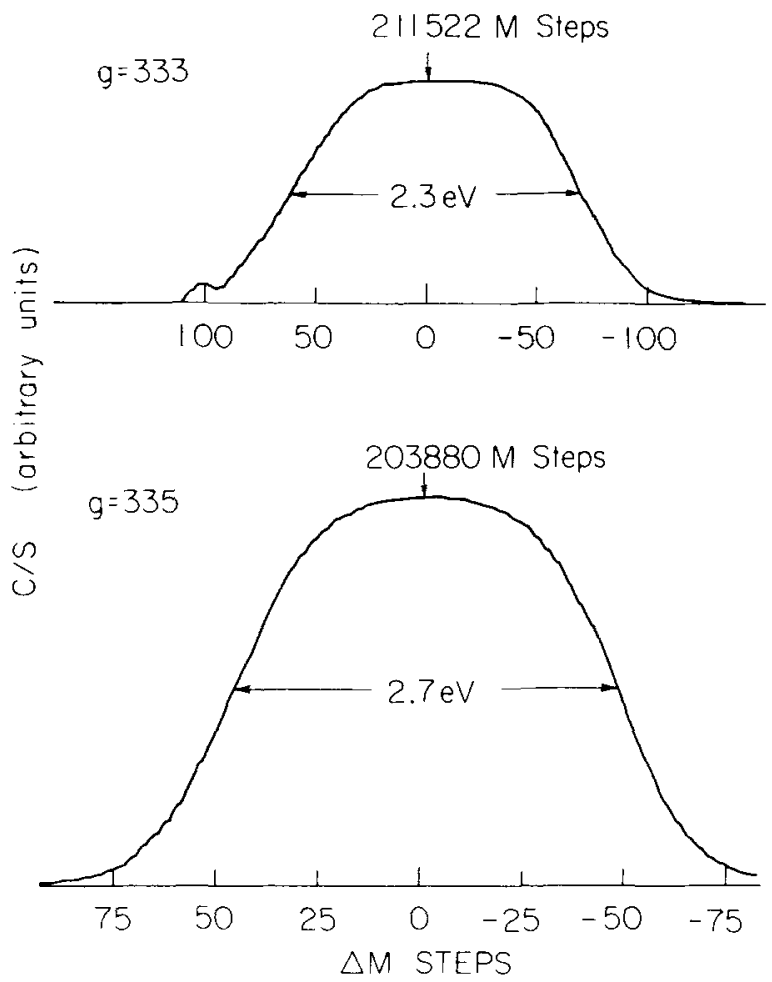

FIG. 5. Diffraction line profiles: (a) $g=(\overline{4} \overline{2} \overline{6})$. (b) $g=(335)$ and (333). The $\mathrm{x}$-ray unit for the $g$ diffraction is $l_{\mathrm{z}}=-\left[1+(\Delta E / E) / \Delta_{\mathrm{gs}}\right]$. For $g=\overline{4} \overline{2} 6$, the origin of $\Delta E / E$ is given for the Bragg angle $\theta_{B}$. However, if the line profile is due to the monochromator diffractions then the $x$-ray unit is $l_{M}$, where $\left(l_{M}+1\right) /\left(l_{s}+1\right)=0.1433$ and the linewidth for each diffraction is: $\Delta \theta=\tan \left(\theta_{M}+c_{M}\right) \Delta E(\mathrm{FWHH}) / 2 E$ $=3.58 \times 10^{-5} \mathrm{rad}=0.74 \mathrm{~s}$ of arc.
$=30 \mathrm{ppm}\left(2 \times 10^{-3} \mathrm{DEG}\right)$. Table I gives the energies evaluated in the neighborhood of the $\mathrm{Cu}$ and $\mathrm{Fe} K$ edges and the Ta $L_{I}$ edge to within an accuracy

$$
\begin{aligned}
\Delta E / E=\Delta S_{M} / S_{M}+\left[\Delta c_{M}\right. & +\theta_{M}(\Delta M / M \\
& \left.\left.+\Delta r_{\theta_{M}} / r_{\theta_{M}}\right)\right] \cot \theta_{M B} .
\end{aligned}
$$

However, the advantage of the method described here lies in the constant monitoring of the energy via the diffraction profiles run with every spectrum. The use of reference markers in the study of the $\mathrm{TaS}_{2}+\mathrm{N}_{2} \mathrm{H}_{4}$ system and $\mathrm{C}+\mathrm{AsF}_{5}$ is shown in Figs. 5, 7, and 8. The actual width of the diffractions was found to depend on the incident flux. For low incident flux (12 mA current) the full width at half-height (FWHH) of the diffraction in Figs. 5 and 7 is $2.3-2.6 \mathrm{eV}$, whereas for high incident flux (65 mA current) we observe a FWHH of $4.4 \mathrm{eV}$ which may be due to poor adjustment of slit widths in the monochromator. ${ }^{6}$

The sharp diffractions $\left[\Delta E(\mathrm{FWHH}) / E=2 \times 10^{-4}\right]$ in Fig. 5 are used as an indication of the reliability of the data. However, this width is considerably greater than the calculated Darwin width for perpendicular polarization ${ }^{6}$ :

$$
\Delta \theta_{g}=4 \delta f(g) \operatorname{csec} 2 \theta / Z=|\Delta E / E|_{D, g} \tan \theta_{B}
$$

or

$$
|\Delta E / E|_{D, g}=2 \delta f(g) \operatorname{csec}^{2} \theta_{B} / Z,
$$

where $\delta=3.8 \times 10^{-6}$ near $11.088 \mathrm{keV}$ for $\mathrm{Si}, f(g)$ is the atomic structure factor $[f(220)=8.70$ and $f(246)$ $\left.=3.68 \pm 0.03^{7}\right]$ and $Z$ is the atomic number. Then the $g=(\overline{2} \overline{4} 6),(\Delta E / E)_{D, g}=1.1 \times 10^{-5}$ is an order of magnitude smaller than the observed full width at half-height (FWHH) in Fig. 5 of $2 \times 10^{-4}$. The Darwin widths are

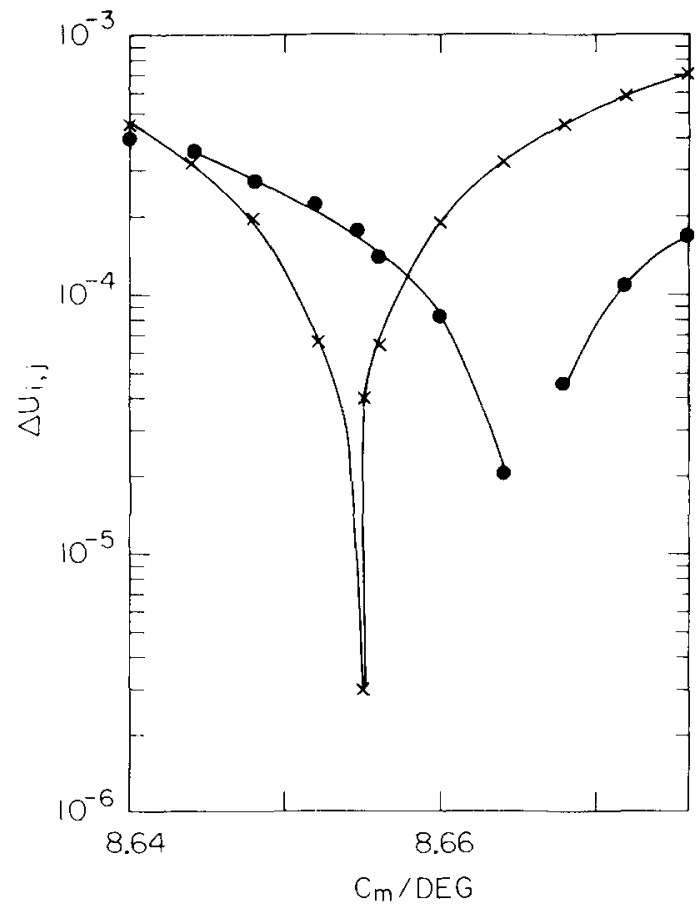

FIG. 6. Dependence of the minimum in $\Delta u_{i, j}$ vs $c_{M}$ on the diff ractions used for the calibration, $\bullet: \Delta u_{3,4}, X: \Delta u_{3,5}$. 
additive. For the two monochromator crystals $(g=220)$ this is greater than that for the reference; i.e., in relation (12) $2(\Delta E / E)_{D, M}=1.08 \times 10^{-4}$ near $11.088 \mathrm{keV}$ is of the same order of magnitude as the observed diffraction width. The physical significance here then is that the reference diffraction actually follows the incident photons' line profile.

The error due to the divergence of the synchrotron beam is determined by the slit height $h \approx 10^{-1} \mathrm{~cm}$ over the distance of the source to slit $\left(R=2 \times 10^{3} \mathrm{~cm}\right)$; i.e., $h / R \approx 4 \times 10^{-5} \mathrm{rad}^{6}$ is less than the Darwin width in this case. However, the diffractions shown in Fig. 5 do not resemble the Darwin profiles evaluated using relation (6.50) of Ref. 6. They are much more symmetric, suggesting that a detailed investigation of diffraction line profiles using synchrotron radiation can yield information on $\delta$ as well as absorption coefficients.

The knowledge of the incident photons' line profile is of some importance in the study of white peaks arising from transitions to bound states below a given edge. In

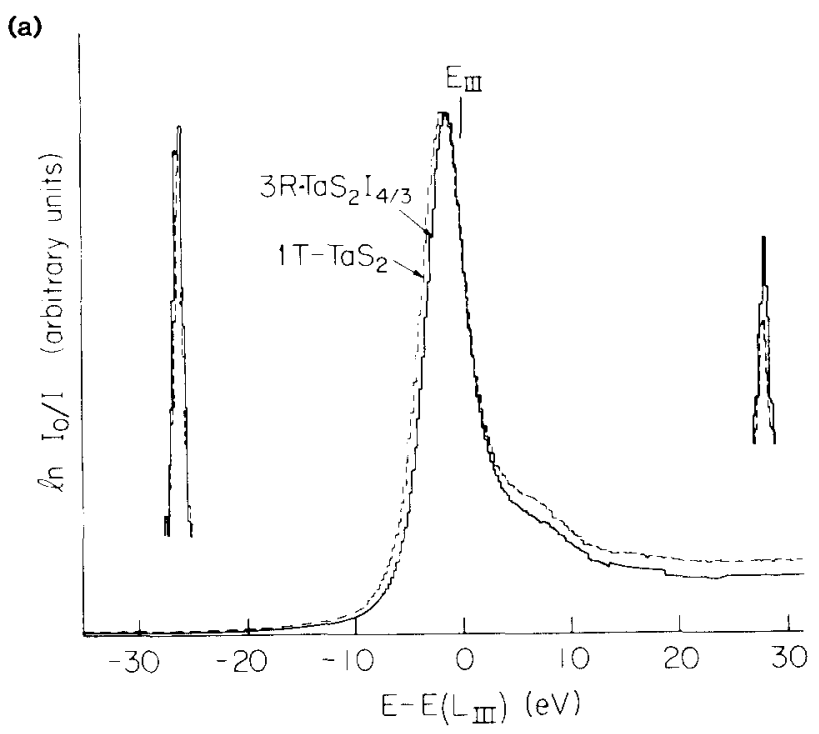

(b)

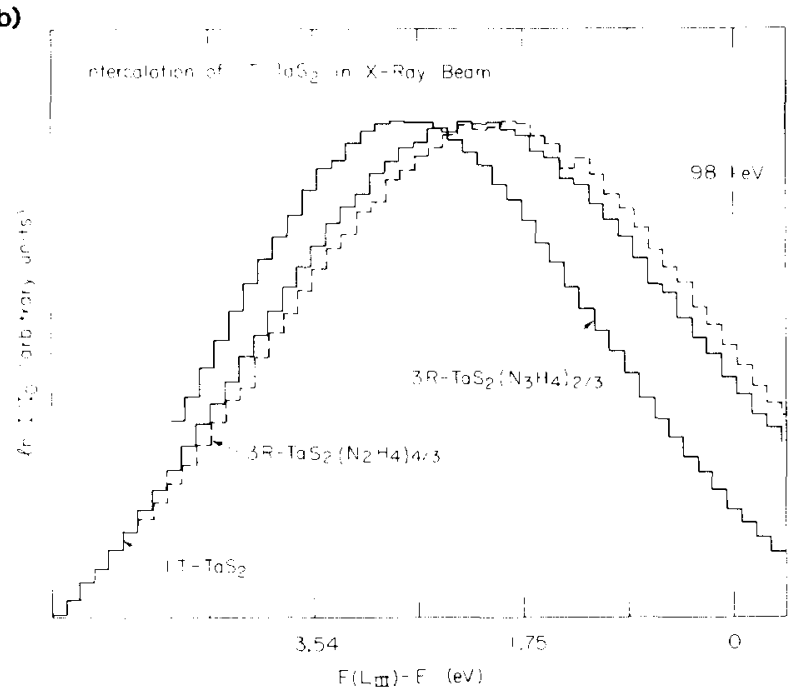

FIG. 7. Observed energy reproducibility: (a) during the intercalation of $\mathrm{TaS}_{2}$ to form $3 R-\mathrm{TaS}_{2} \cdot \mathrm{I}_{4 / 3}$; (b) comparison of edge energies for the three compounds in relation (6).

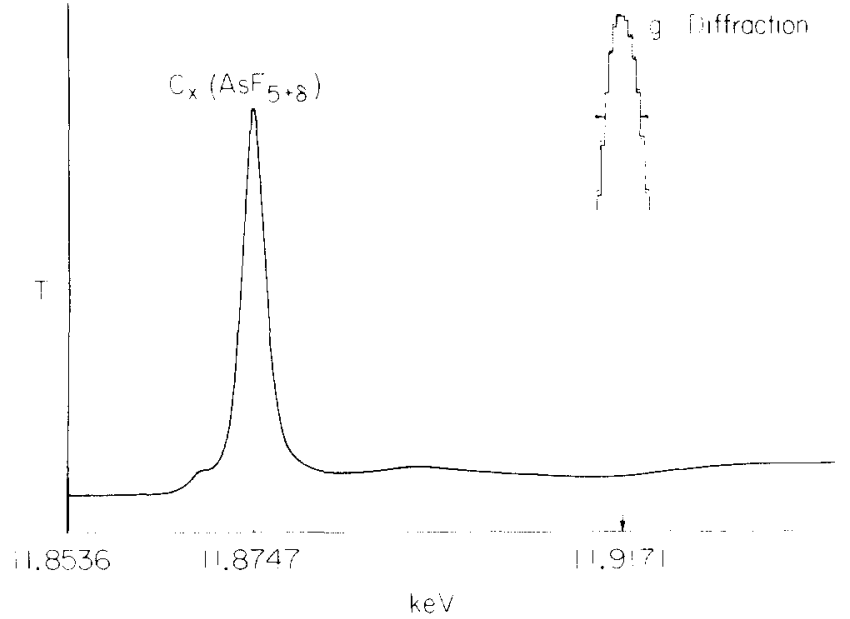

FIG. 8. Observed energy reproducibility near the As $K$ edge. Here two consecutive runs are superimposed.

the study of IT-TaS $\mathrm{T}_{2}$ we can measure changes in the line profile due to intercalation with $\mathrm{N}_{2} \mathrm{H}_{4}$. The sharp reference diffractions shown in Fig. $6(2.6 \mathrm{eV} \mathrm{FWHH})$ ensure that the incident photons' line profiles are narrower than the width of the measured absorption peaks near the Ta- $L$ edges. The shifts in energy shown in Fig. 6 must be related to changes in the structure due to intercalation. ${ }^{89}$ Here the intercalation reaction

$$
\begin{aligned}
\text { IT }-\mathrm{TaS}_{2} \underset{13 \text { Torr }}{\stackrel{+4 / 3 \mathrm{~N}_{2} \mathrm{H}_{4}}{\rightleftarrows}} 3 R-\mathrm{TaS}_{2}\left(\mathrm{~N}_{2} \mathrm{H}_{4}\right)_{4 / 3} \underset{10^{-3} \text { Torr }}{\stackrel{-2 / 3 \mathrm{~N}_{2} \mathrm{H}_{4}}{\rightleftarrows}} \\
3 R-\mathrm{TaS}_{2}\left(\mathrm{~N}_{2} \mathrm{H}_{4}\right)_{2 / 3}
\end{aligned}
$$

introduces commensurate periodic lattice distortions into IT-TaS ${ }_{2}$ which have been associated with multiple valence states. The accurate measurements shown in Fig. 7 indicate the $\mathrm{Ta}$ valence is increased when two layers of $\mathrm{N}_{2} \mathrm{H}_{4}$ are intercalculated in between the $\mathrm{TaS}_{2}$ layers but that it is decreased when only one layer of $\mathrm{N}_{2} \mathrm{H}_{4}$ remains in between the layers. These fractional valence changes must be associated with charge transfer from $\mathrm{N}_{2} \mathrm{H}_{4}$ to the $\mathrm{TaS}_{2}$ layers.

The width of the white line profile near the As $K$ edge in $\mathrm{C}$ (graphite) $+\mathrm{AsF}_{5}$ shown in Fig. 8 is of the same order of magnitude as the FWHH of the diffraction of $4.4 \mathrm{eV}$. As a result one cannot determine the actual absorption profile because we suspect the diffraction width is caused by the width of the incident photons' line profile. This poor resolution may be due to heating of the monochromator crystals by the incident flux and/or poor slit adjustment. However, the reference diffraction is the only possible way to establish the stability and condition of the incident photons.

\section{APPENDIX}

The $n(111)$ diffractions used to calibrate $c_{M}$ in the three-crystal spectrometer are insensitive to any misalignment in $\mathbf{g}_{0}, \mathbf{g}_{x}$, and $\mathbf{g}_{\boldsymbol{y}}$. However, since other $\mathbf{g}$ $=(h k l)$ diffractions may be used to monitor the energy near an edge being measured, it is important to determine 
any alignment crrors. Let

$$
\begin{aligned}
& \mathbf{g}_{x}=(\overline{1} \overline{1} 2)+\mathbf{x} ; \quad \mathbf{g}_{y}=(1 \overline{1} 0)+\mathbf{y} ; \\
& \mathbf{g}_{0}=(111)+\mathbf{z},
\end{aligned}
$$

where $\mathbf{x}, \mathbf{y}, \mathbf{z}$ represent alignment errors which are related by the orthogonality relations:

$$
\begin{aligned}
\mathbf{g}_{x} \cdot \mathbf{g}_{0} \cong \mathbf{x} \cdot \mathbf{g}_{0}+\mathbf{z} \cdot \mathbf{g}_{x} \cong \mathbf{g}_{y} \cdot \mathbf{g}_{0} \cong \mathbf{y} \cdot \mathbf{g}_{0}+\mathbf{z} \cdot \mathbf{g}_{y} \\
\cong \mathbf{g}_{x} \cdot \mathbf{g}_{y} \cong \mathbf{x} \cdot \mathbf{g}_{y}+\mathbf{y} \cdot \mathbf{g}_{x} \cong 0 .
\end{aligned}
$$

The diffraction energy is evaluated in (2) using the ratio $\sin \theta_{g} / g$ and the error introduced by a misalignment (Al) into the energy is:

$$
\Delta_{a} g=\Delta E_{g} / E_{g}=-\left(\bar{\theta}_{g}-\theta_{g}\right) \cot \theta_{g},
$$

where $\bar{\theta}_{g}$ is the true value and $\theta_{g}$ assumes $\mathbf{x}=\mathbf{y}=\mathbf{z}$ $=0$. Then using $\left(2^{\prime}\right)$ :

$$
\begin{aligned}
\Delta_{a} g= & -\left(\sin \bar{\theta}_{g}-\sin \theta_{g}\right) / \sin \theta_{g} \\
= & -\left\{3^{-3 / 2} \sin \theta_{0}\left[3 z \cdot \mathbf{g}-\left(\mathbf{z} \cdot \mathbf{g}_{0}\right)\left(\mathbf{g} \cdot \mathbf{g}_{0}\right)\right]+6^{-3 / 2}\right. \\
& \left.\times \cos \theta_{0}\left[6 \mathbf{x} \cdot \mathbf{g}-\left(\mathbf{g}_{x} \cdot \mathbf{x}\right)\left(\mathbf{g}_{x} \cdot \mathbf{g}\right)\right]\right\} /\left(g \sin \theta_{g}\right) .
\end{aligned}
$$

The cubic symmetry of the crystal allows us to simplify the error analysis. Here:

$$
\begin{aligned}
\Delta_{a} n n n= & -18^{-1 / 2} \mathbf{x} \cdot \mathbf{g}_{0} \cot \theta_{0} \equiv \Delta_{a}^{0}, \\
\Delta_{a} h h l=\Delta_{a}^{0}[(2 h+l) & \\
& \left.-2^{1 / 2}(l-h) \tan \theta_{0}\right] \sin \theta_{0} /\left(g g_{0} \sin \theta_{g}\right),
\end{aligned}
$$

e.g., near $\theta_{0}=43^{\circ}$ we obtain

$$
\Delta E_{333} / E_{333}-\Delta E_{335} / E_{335}=0.41 \Delta_{a}^{0}
$$

and

$$
\Delta E_{333} / E_{333}-\Delta E_{0012} / E_{0012}=1.1 \Delta_{a}^{0} .
$$

When $\theta_{0}$ is evaluated assuming $\Delta E_{n n n}=0$, then relations (A5) indicate that the diffractions $g=(h h l)$ should be predicted to within $\Delta_{a}^{0}=3.7 \times 10^{-3}$ in Table I.

\section{ACKNOWLEDGMENTS}

This work was done under the auspices of NSF DMR 7820577 and proposals $312 \mathrm{M}$ and $512 \mathrm{M}$ at SSRL. The facilities at SSRL are supported under NSF contract DMR 77-27489 in cooperation with the U.S. Department of Energy. We are grateful to J. R. Salem, E. Grove, and T. Rowe for the Si crystals, R. Ott for machine shop work, E. Langone for computer analysis, and S. Wootton, S. Brown, and H. Stein for typing.

${ }^{1}$ J. V. Acrivos, K. Hathaway, A. Robertson, A. Thompson, and M. P. Klein, J. Phys. Chem. 84, 1206 (1980); and J. Phys. C 14, 1557 ( I 981$)$

${ }^{2}$ J. M. Tranquada, R. Ingalls, J. E. Whitmore, and E. D. Crozier,

Seventh Annual SSRL Meeting, SSRL Report 80/05, p. 74.

${ }^{3}$ J. B. Boyce, R. M. Martin and J. W. Allen, Seventh Annual SSRL Meeting, SSRL Report 80/05, p. 128.

${ }^{4}$ J. V. Acrivos, S. Parkin, J. Code, J. Reynolds, K. Hathaway, H. Kurasaki, and E. Marseglia, Seventh Annual SSRL Meeting, SSRL Report 80/05, p. 79; J. Phys. C. 14, L349 (1981).

${ }^{5}$ K. E. Falk, D. B. Goodin, M. P. Klein, and T. Wydrzynski, Seventh Annual SSRL meeting, SSRL Report 79/05.

${ }^{6}$ A. H. Compton and S. K. Allison, $X$-Rays in Theory and Experiment (Van Nostrand, Princeton, New Jersey, 1963) Chap. VI; G. S. Brown and S. Doniach, Synchrotron Radiation, $\mathrm{H}$. Winnick and S. Doniach (Plenum, New York, 1981)

${ }^{7}$ B. Dawson. Proc. R. Soc. London 298A, 379 (1967).

$8 \mathrm{~J}$. V. Acrivos, Intercalated Layer Materials, edited by F. Levy (Reidel, Dordrecht, Holland, 1979), Vol. VI.

${ }^{9}$ G. Tatlock and J. V. Acrivos, Philos. Mag. 38, 81 (1978). 